\title{
LA EDUCACIÓN AMBIENTAL PARA LA SUSTENTABILIDAD Y SU CONTRIBUCIÓN AL APRENDIZAJE HOLÍSTICO EN LA FACULTAD DE ARQUITECTURA, URBANISMO Y ARTES, UNIVERSIDAD NACIONAL DE INGENIERÍA, LIMA, PERÚ ${ }^{1}$
}

\section{ENVIRONMENTAL EDUCATION FOR SUSTAINABILITY AND ITS CONTRIBUTION TO HOLISTIC LEARNING AT THE FACULTY OF ARCHITECTURE, URBANISM AND ARTS, NATIONAL UNIVERSITY OF ENGINEERING, LIMA, PERÚ}

DOI: https://doi.org/10.47796/ra.2021i19.552

SHIRLEY EMPERATRIZ CHILET CAMA

Universidad Nacional de Ingeniería, Lima - Perú

https://orcid.org/0000-0002-6330-7240

schilet@uni.edu.pe

RESUMEN

El objetivo de este estudio es explicar cómo la educación ambiental para la sustentabilidad es importante en la formación académica estudiantil para un aprendizaje con visión holística en la Facultad de Arquitectura, Urbanismo y Artes (FAUA) de la Universidad Nacional de Ingeniería (UNI) en Lima. La investigación, de tipo básica, tuvo un enfoque cuantitativo, correlacional causal con un diseño no experimental. La población estuvo conformada por 80 docentes y 100 estudiantes. El instrumento aplicado fue el cuestionario. Se evidencia que los docentes, en menor escala, incorporan en sus diferentes asignaturas la educación ambiental con contenidos actitudinales y axiológicos que contribuyan a la formación de valores y actitudes de convivencia; pero sí consideran que la educación ambiental es importante para la sustentabilidad con visión holística.

PALABRAS CLAVE: educación ambiental, sostenibilidad, aprendizaje holístico.

\section{ABSTRACT}

The objective of this study is to explain how environmental education for sustainability is important in student academic training for learning with a holistic vision at the Faculty of Architecture, Urbanism and Arts of the National University of Engineering in Lima. The basic research had a quantitative, causal correlational approach with a non-experimental design. The population consisted of 80 teachers and 100 students. The instrument applied was the questionnaire. It is evidenced that teachers, on a smaller scale, incorporate environmental education in their different subjects with attitudinal and axiological contents that contribute to the formation of values and attitudes of coexistence; but they do consider that environmental education is important for sustainability with a holistic vision.

KEYWORDS: environmental education, sustainability, holistic learning.

\footnotetext{
El presente artículo es resultado de una investigación doctoral en la especialidad de Medio Ambiente y Desarrollo Sostenible.
} 


\section{INTRODUCCIÓN}

Cóndor (2016) sostiene que es necesario implementar acciones para incorporar la dimensión ambiental en las principales funciones de la universidad: la investigación, la extensión universitaria, la proyección social. Por su parte, Pernía (2016) refiere que la sustentabilidad depende de la interacción de todas las dimensiones y las identifica como la ecológica, la social, la institucional, la económica y la tecnológica, en torno a las cuales se debe articular un equilibrio. Esquerra, Gil y Márquez (2016) explican que los procesos de ambientalización constituyen una nueva filosofía de vida. En la FAUA-UNI, no se considera la educación ambiental como eje transversal en la malla curricular. El currículo de las diferentes asignaturas no está basado en la interdisciplinariedad, no se da la interacción de las diversas disciplinas que promuevan la generación de nuevos conocimientos; tampoco existe una etapa superior de integración disciplinar como es la transdisciplinariedad, que se traduce en una carencia de un conocimiento global y multidimensional de los aspectos de la realidad, puesto que no son tratados en las áreas académicas, lo cual es fundamental en la educación ambiental. Entonces, ¿la educación ambiental para la sustentabilidad es importante en la formación académica estudiantil para un aprendizaje con visión holística en la Facultad de Arquitectura, Urbanismo y Artes de la Universidad Nacional de Ingeniería? Esta investigación es importante porque considera a la educación ambiental como el pilar fundamental para alcanzar e desarrollo sostenible, y está orientada a formar ciudadanos activos y comprometidos en la construcción de una sociedad más justa, democrática y solidaria. Es por ello que la Universidad es un espacio privilegiado para llevar adelante acciones que, partiendo de realidades complejas, tengan como horizonte un desarrollo económicamente viable, justo y ecológicamente equilibrado.
El objetivo principal de la investigación fue conocer si los docentes incorporaron la educación ambiental en sus diferentes asignaturas con contenidos actitudinales y axiológicos que contribuyan a la formación de valores y actitudes de convivencia, respeto y de comportamiento del individuo con el medio; si los docentes consideraron que es importante la interacción de diversas disciplinas en la formación académica estudiantil para lograr una transmisión activa de nuevos conocimientos, desarrollo físico, cognitivo, espiritual y de satisfacción; si los docentes consideraron que es importante el conocimiento global y multidimensional delos diversos aspectos de la realidad en la formación académica estudiantil para lograr tener una conciencia ambiental y compromiso social y ciudadano.

Como hipótesis central se planteó que la educación ambiental para la sustentabilidad es importante en la formación académica estudiantil para un aprendizaje con visión holística, basado en la integración de conocimientos en la FAUA-UNI.

\section{MATERIALESY MÉTODOS}

La presente investigación es básica y tuvo un enfoque cuantitativo, correlacional causal, con un diseño no experimental transversal. La población del estudio estuvo conformada por 80 docentes y 100 estudiantes de la FAUA-UNI. De ella, se extrajo una muestra de 36 docentes y 46 estudiantes matriculados en el ciclo académico 2019-1 de la Facultad.

Para el diseño del instrumento, se ha tomado en cuenta los objetivos, las hipótesis y las variables. El instrumento aplicado ha sido un cuestionario que guarda la confiabilidad, validez y objetividad. Este cuestionario (con preguntas 
cerradas) fue aplicado a docentes y estudiantes. El cuestionario, que contiene 20 preguntas para docentes y 117 afirmaciones para estudiantes, versó sobre la educación ambiental para la sustentabilidad en el currículo (en el caso de los docentes), y sobre el aprendizaje holístico, valores y actitudes (en el caso de estudiantes) para conocer su desarrollo humano.

Otro instrumento de medición o de recolección de datos que se aplicó a los estudiantes fue la escala tipo Likert para medir sus actitudes. Sin embargo, hay que tomar en cuenta que la actitud por su naturaleza es subjetiva, no es susceptible de observación directa, por tanto, ha de inferirse de la conducta manifiesta, en este caso, a través de la expresión verbal de los estudiantes de investigación; ello permitirá reflejar actitudes positivas o negativas, favorables y desfavorables, respectivamente.

\section{RESULTADOS}

Tabla 1. Distribución de 36 Docentes de la FAUA - UNI, según su Opinión Sí/No es importante la Incorporación de la Educación Ambiental para la Sustentabilidad en la Formación Académica.

\begin{tabular}{c|c|c|c}
\hline Niveles & Frecuencias & $\begin{array}{c}\text { Porcentaje } \\
(\mathbf{\%})\end{array}$ & $\begin{array}{c}\text { Porcentaje } \\
\text { Acumulado } \\
(\%)\end{array}$ \\
\hline No & 2 & 5.6 & 5.6 \\
Sí & 34 & 94.4 & 100.0 \\
Total & 36 & 100.0 & \\
\hline
\end{tabular}

Fuente: Elaboración propia

Figura 1. Distribución de 36 docentes de la FAUA - UNI, según su opinión Si/No es importante la incorporación de la Educación Ambiental para la Sustentabilidad en la formación académica.

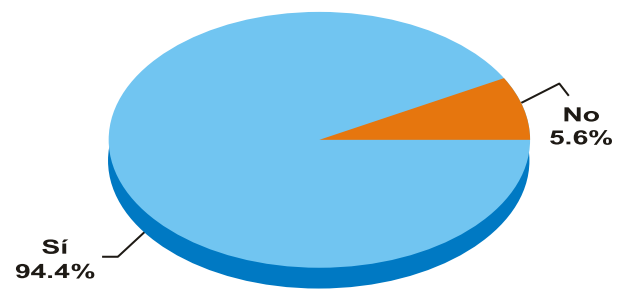

Fuente: Elaboración propia
Para determinar la validez y confiabilidad del instrumento de medición de las dos variables de estudio, así como sus dimensiones, se realizaron dos muestras pilotos a docentes y estudiantes de la FAUA-UNI. Asimismo, se hizo uso del coeficiente Alfa Cronbach para los ítems medidos en escala de Likert; mientras que para aquellos ítems de tipo dicotómico se usó el método KR- 20.

Para el procesamiento de los datos se ha usado la herramienta estadística SPSS 23.0 (Statistical Product and Service Solutions). Por otro lado, el análisis de los datos comprendió dos partes. El primero, un análisis descriptivo de las variables de estudio y sus respectivas dimensiones mediante técnicas de Estadística Descriptiva; la segunda, la aplicación de técnicas de Estadística Inferencial para contrastar las hipótesis planteadas mediante pruebas hipótesis para una proporción.

En la Tabla 1 y Figura 1, se observa que el 94.4\% de los docentes de la FAUA-UNI consideran que sí es importante la incorporación de la educación ambiental para la sustentabilidad en la formación académica de los estudiantes de esta Facultad, mientras que el $5.6 \%$, lo considera no importante.

\section{Dimensión Eje Transversal}

Tabla 2. Distribución de 36 Docentes de la FAUA - UNI, según su Opinión Sí/No es Importante la Incorporación de la Educación Ambiental para la Sustentabilidad como Eje Transversal en la Formación Académica.

\begin{tabular}{c|c|c|c}
\hline Niveles & Frecuencias & $\begin{array}{c}\text { Porcentaje } \\
(\%)\end{array}$ & $\begin{array}{c}\text { Porcentaje } \\
\text { Acumulado } \\
(\%)\end{array}$ \\
\hline No & 2 & 5.6 & 5.6 \\
Sí & 34 & 94.4 & 100.0 \\
Total & 36 & 100.0 & \\
\hline
\end{tabular}

Fuente: Elaboración propia 
Figura 2. Distribución de 36 Docentes de la FAUA - UNI, según su Opinión Sí/No es Importante la Incorporación de la Educación Ambiental para la Sustentabilidad como Eje Transversal en la Formación Académica.

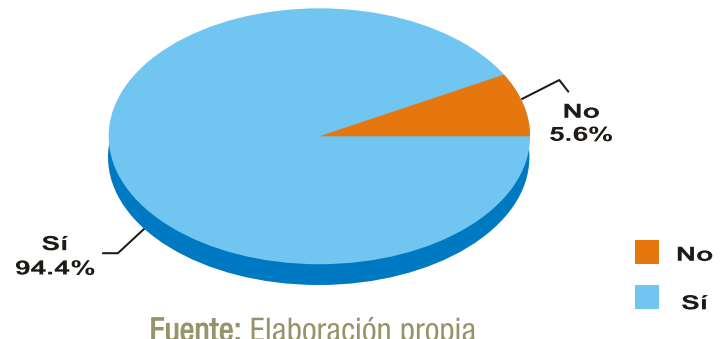

Se observa, en la Tabla 2 y Figura 2, que el 94.4\% de los docentes de Ia FAUA-UNI consideran que sí es importante la incorporación de la educación ambiental para la sustentabilidad como eje transversal en la formación académica de los estudiantes de esta Facultad; el $5.6 \%$ de los encuestados consideran que no es importante.

Tabla 3. Distribución de 36 Docentes de la FAUA - UNI, según su Opinión Sí/No es Importante la Inclusión de la Dimensión Ambiental en la Formación Académica

\begin{tabular}{c|c|c|c}
\hline Niveles & Frecuencias & $\begin{array}{c}\text { Porcentaje } \\
\text { (\%) }\end{array}$ & $\begin{array}{c}\text { Porcentaje } \\
\text { Acumulado } \\
(\%)\end{array}$ \\
\hline No & 14 & 38.9 & 38.9 \\
Sí & 22 & 61.1 & 100.0 \\
Total & 36 & 100.0 & \\
\hline
\end{tabular}

Fuente: Elaboración propia

Figura 3. Distribución de 36 Docentes de la FAUA - UNI, según su Opinión Sí/No es Importante la Inclusión de la Dimensión Ambiental en la Formación Académica

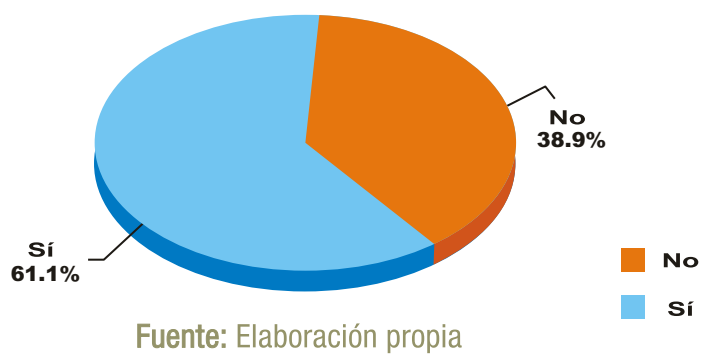

En la Tabla 3 y Figura 3 se puede apreciar que el $61.1 \%$ de los docentes de la Facultad de Arquitectura de la Universidad Nacional de Ingeniería consideran que sí es importante la inclusión de la dimensión ambiental en la formación académica de los estudiantes de esta Facultad, mientras que el $38.9 \%$ de los docentes considera que no lo es.

Tabla 4. Distribución de 36 Docentes de la FAUA - UNI, según su Opinión Sí/No es Importante la incorporación de Contenidos Actitudinales y Axiológicos en la Formación Académica

\begin{tabular}{c|c|c|c}
\hline Niveles & Frecuencias & $\begin{array}{c}\text { Porcentaje } \\
(\mathbf{\%})\end{array}$ & $\begin{array}{c}\text { Porcentaje } \\
\text { Acumulado } \\
(\%)\end{array}$ \\
\hline No & 6 & 16.7 & 16.7 \\
Sí & 30 & 83.3 & 100.0 \\
Total & 36 & 100.0 & \\
\hline
\end{tabular}

Fuente: Elaboración propia

Figura 4. Distribución de 36 Docentes de la FAUA - UNI, según su Opinión Sí/No es Importante la Incorporación de Contenidos Actitudinales y Axiológicos en la Formación Académica

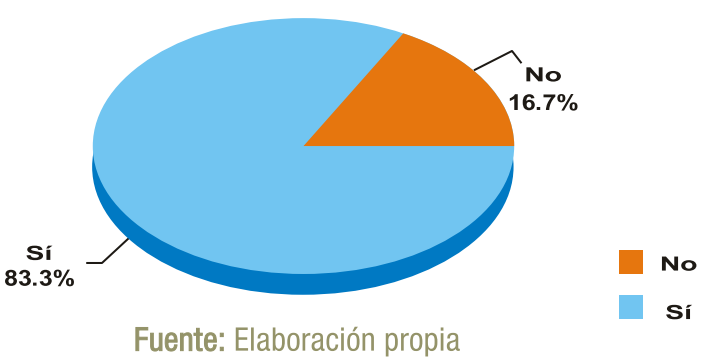

La Tabla 4 y Figura 4 muestra que el 83.3\% de los docentes de la FAUA de la Universidad Nacional de Ingeniería considera que la incorporación de contenidos actitudinales y axiológicos en la formación académica de los estudiantes de esta Facultad sí es importante; en cambio, el $16.7 \%$, no lo considera así.

Tabla 5. Distribución de 36 Docentes de la FAUA - UNI, según Nivel de Importancia de Tener Formación Académica, en Educación Ambiental para la Sustentabilidad.

\begin{tabular}{l|c|c|c}
\hline \multicolumn{1}{c|}{ Niveles } & Frecuencias & $\begin{array}{c}\text { Porcentaje } \\
\mathbf{( \% )}\end{array}$ & $\begin{array}{c}\text { Porcentaje } \\
\text { Acumulado } \\
\mathbf{( \% )}\end{array}$ \\
\hline Poco Importante & 2 & 5.6 & 5.6 \\
Indiferente & 8 & 22.2 & 27.8 \\
Importante & 26 & 72.2 & 100.0 \\
Total & 36 & 100.0 & \\
\hline
\end{tabular}

Fuente: Elaboración propia 
Figura 5. Distribución de 36 Docentes de la FAUA - UNI, según nivel de Importancia de tener Formación Académica en Educación Ambiental para la Sostenibilidad

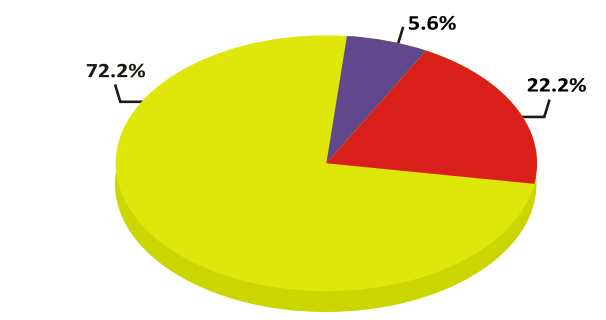

Poco Importante

Indiferente a Importante

Fuente: Elaboración propia

En la Tabla 5 y Figura 5 se puede apreciar los niveles de importancia respecto de tener incorporado en la formación académica la educación ambiental para la sustentabilidad: el $72.2 \%$ de los docentes de la FAUA de la Universidad Nacional de Ingeniería lo considera importante; el $22.2 \%$, le es indiferente, y el $5.6 \%$, poco importante.

\section{Dimensión Enfoque Interdisciplinario}

Tabla 6. Distribución de 36 Docentes de la FAUA- UNI, según su Opinión Sí/No es Importante la Incorporación de la Educación Ambiental como Enfoque Interdisciplinario en la Formación Académica

\begin{tabular}{c|c|c|c}
\hline Niveles & Frecuencias & $\begin{array}{c}\text { Porcentaje } \\
\text { (\%) }\end{array}$ & $\begin{array}{c}\text { Porcentaje } \\
\text { Acumulado } \\
\text { (\%) }\end{array}$ \\
\hline No & 1 & 2.8 & 2.8 \\
Sí & 35 & 97.2 & 100.0 \\
Total & 36 & 100.0 & \\
\hline
\end{tabular}

Fuente: Elaboración propia

Figura 6. Distribución de 36 Docentes de la FAUA - UNI, según su Opinión Sí/No es Importante la Incorporación de la Educación Ambiental como Enfoque Interdisciplinario en la Formación Académica

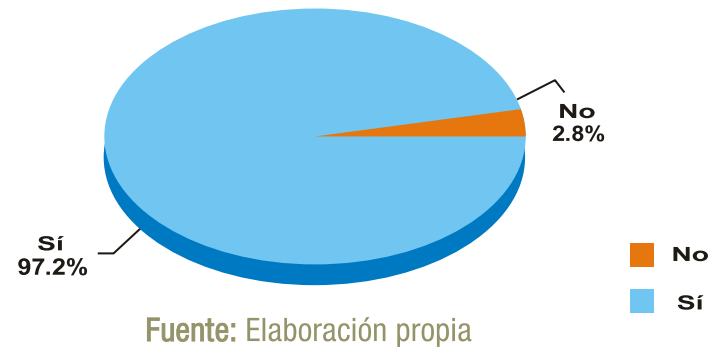

Se observa de la Tabla 6 y Figura 6, que el 97.2\%, de los docentes de la Facultad de Arquitectura, Urbanismo y Artes de la Universidad Nacional de Ingeniería consideran que sí es importante la incorporación de la Educación Ambiental para la Sustentabilidad como Enfoque Interdisciplinario en la formación académica de los estudiantes de esta facultad; $y$ el $2.8 \%$, consideran que no es importante.

\section{Dimensión Enfoque Transdisciplinario}

Tabla 7. Distribución de 36 Docentes de la FAUA - UNI, según su Opinión Sí/No es Importante la Incorporación de la Educación Ambiental como Enfoque

Transdisciplinario en la Formación Académica

\begin{tabular}{c|c|c|c}
\hline Niveles & Frecuencias & $\begin{array}{c}\text { Porcentaje } \\
(\mathbf{\%})\end{array}$ & $\begin{array}{c}\text { Porcentaje } \\
\text { Acumulado } \\
(\mathbf{\%})\end{array}$ \\
\hline No & 10 & 27.8 & 27.8 \\
Sí & 26 & 72.2 & 100.0 \\
Total & 36 & 100.0 & \\
\hline
\end{tabular}

Fuente: Elaboración propia

Figura 7. Distribución de 36 Docentes de la FAUA - UNI, según su Opinión Sí/No es Importante la Incorporación de la Educación Ambiental como Enfoque

Transdisciplinario en la Formación Académica

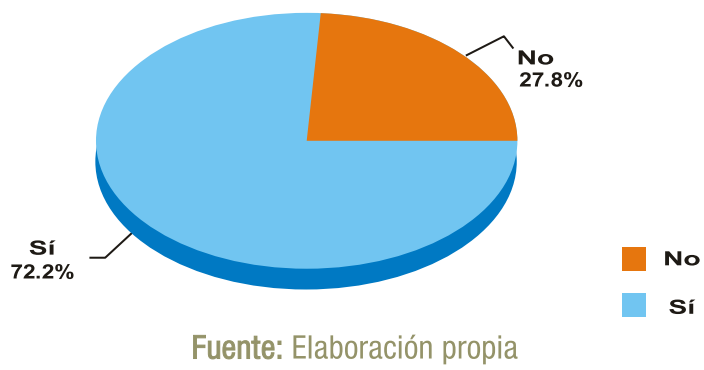

La Tabla 7 y Figura 7 permite observar que el $72.2 \%$ de los docentes de la FAUA - UNI considera importante la incorporación de la educación ambiental para la sustentabilidad como enfoque transdisciplinario en la formación académica de los estudiantes de esta Facultad; mientras que el $27.8 \%$ considera no importante. 
Tabla 8. Distribución de 46 Estudiantes de la FAUA - UNI, según Nivel de Importancia del Aprendizaje Holístico en la Formación Académica

\begin{tabular}{l|c|c|c}
\hline \multicolumn{1}{c|}{ Niveles } & Frecuencias & $\begin{array}{c}\text { Porcentaje } \\
(\mathbf{\%})\end{array}$ & $\begin{array}{c}\text { Porcentaje } \\
\text { Acumulado } \\
(\mathbf{\%})\end{array}$ \\
\hline Mediana Importancia & 10 & 21.7 & 21.7 \\
Máxima Importancia & 36 & 78.3 & 100.0 \\
Total & 46 & 100.0 & \\
\hline
\end{tabular}

Fuente: Elaboración propia

Figura 8. Distribución de 46 Estudiantes de la FAUA - UNI, según Nivel de Importancia del Aprendizaje Holístico en la Formación Académica

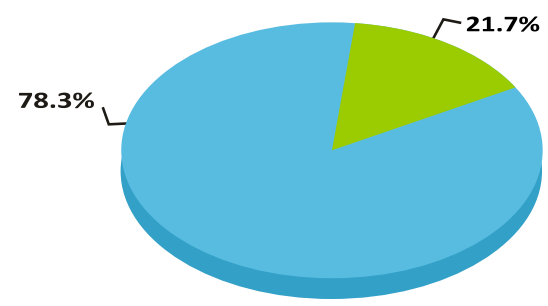

Mediana Importancia $\square$ Máxima importancia

Como se aprecia en la Tabla 8 y Figura 8 , respecto a los niveles de importancia respecto al aprendizaje holístico en la formación académica de los estudiantes de la FAUA de la Universidad Nacional de Ingeniería, el $78.3 \%$ de los encuestados considera que esta es de máxima importancia; mientras que el $21.7 \%$ le otorga mediana importancia.

\section{Dimensión Interpersonal}

Tabla 9. Distribución de 46 Estudiantes de la

Facultad de Arquitectura de la UNI, según

Nivel de Importancia del Aprendizaje Holístico Referido a lo Interpersonal en la Formación

\begin{tabular}{l|c|c|c}
\hline \multicolumn{1}{c|}{ Niveles } & Frecuencias & $\begin{array}{c}\text { Porcentaje } \\
\mathbf{( \% )}\end{array}$ & $\begin{array}{c}\text { Porcentaje } \\
\text { Acumulado } \\
(\mathbf{\%})\end{array}$ \\
\hline Mediana Importancia & 10 & 21.7 & 21.7 \\
Máxima Importancia & 36 & 78.3 & 100.0 \\
Total & 46 & 100.0 & \\
\hline
\end{tabular}

Fuente: Elaboración propia
Figura 9. Distribución de 46 Estudiantes de la FAUA - UNI, según Nivel de Importancia del Aprendizaje Holístico Referido a lo Interpersonal en la Formación Académica

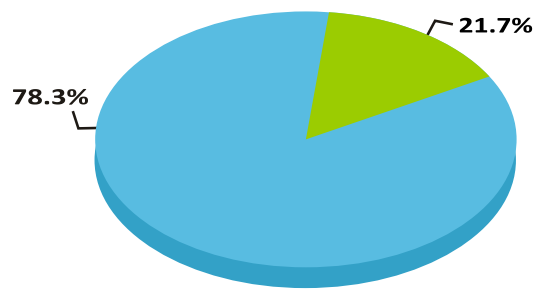

Mediana Importancia

Máxima importancia

En la Tabla 9 y Figura 9, se puede apreciar los niveles de importancia respecto al aprendizaje holístico referido a lo interpersonal en la formación académica de los estudiantes de la FAUA de la Universidad Nacional de Ingeniería: el $78.3 \%$ de los encuestados considera que es de máxima importancia; el $21.7 \%$, de mediana importancia.

Tabla 10. Distribución de 46 Estudiantes de la FAUA - UNI, según Nivel de Importancia del Aprendizaje Holístico Referido a Ios Valores de Convivencia en la Formación Académica

\begin{tabular}{l|c|c|c}
\hline Niveles & Frecuencias & $\begin{array}{c}\text { Porcentaje } \\
\mathbf{( \% )}\end{array}$ & $\begin{array}{c}\text { Porcentaje } \\
\text { Acumulado } \\
\mathbf{( \% )}\end{array}$ \\
\hline Mediana Importancia & 9 & 19.6 & 19.6 \\
Bastante Importancia & 33 & 71.7 & 91.3 \\
Máxima Importancia & 4 & 8.7 & 100.0 \\
Total & 46 & 100.0 & \\
\hline
\end{tabular}

Fuente: Elaboración propia

Figura 10. Distribución de 46 Estudiantes de la FAUA - UNI, según Nivel de Importancia del Aprendizaje Holístico Referido a los Valores de Convivencia en la Formación Académica

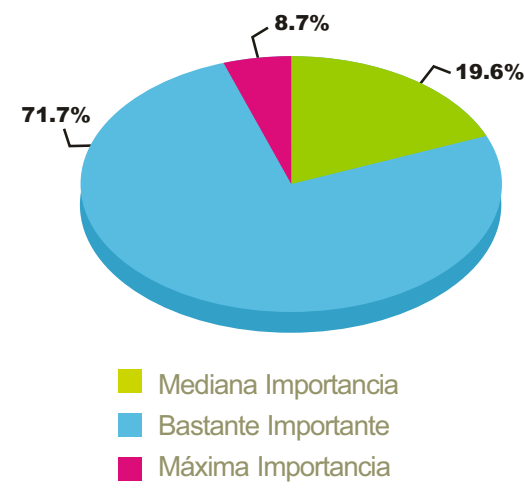

Fuente: Elaboración propia 
Respecto al aprendizaje holístico referido a los valores de convivencia en la formación académica de los estudiantes de la FAUA de la Universidad Nacional de Ingeniería, se observa, en la Tabla 10 y Figura 10, que el 8.7\% de los encuestados lo considera de máxima importancia; el $71.7 \%$, bastante importante, yel $19.6 \%$, de mediana importancia.

Tabla 11. Distribución de 46 Estudiantes de la Facultad de Arquitectura de la UNI, según su Nivel de Actitudes de Respeto a la Persona

\begin{tabular}{l|c|c|c}
\hline \multicolumn{1}{c|}{ Niveles } & Frecuencias & $\begin{array}{c}\text { Porcentaje } \\
\mathbf{( \% )}\end{array}$ & $\begin{array}{c}\text { Porcentaje } \\
\text { Acumulado } \\
(\%)\end{array}$ \\
\hline Regular & 1 & 2.2 & 2.2 \\
Buena & 18 & 39.1 & 41.3 \\
Muy Buena & 27 & 58.7 & 100.0 \\
Total & 46 & 100.0 & \\
\hline
\end{tabular}

Fuente: Elaboración propia

Figura 11. Distribución de 46 Estudiantes de la FAUA - UNI, según su Nivel de Actitudes de Respeto a la Persona

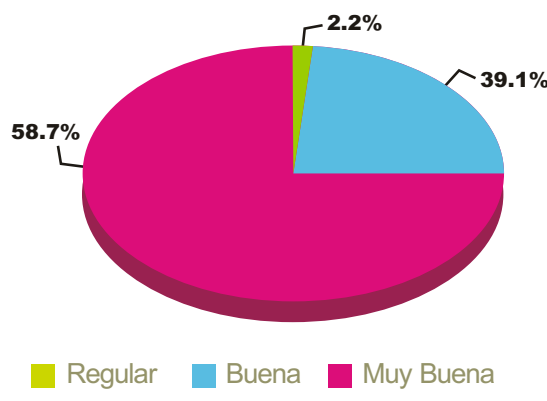

Fuente: Elaboración propia

Se observa en la Tabla 11 y Figura 11 que, respecto a sus niveles de actitudes de respeto a la persona de los estudiantes de la Facultad de Arquitectura, Urbanismo y Artes de la Universidad Nacional de Ingeniería, el 58.7\% de los encuestados consideran que su actitud es muy buena; el $39.1 \%$, buena, y el $2.2 \%$, regular.

Tabla 12. Distribución de 46 Estudiantes de la FAUA - UNI, según su Nivel de Actitudes de Aprender a Convivir

\begin{tabular}{l|c|c|c}
\hline \multicolumn{1}{c|}{ Niveles } & Frecuencias & $\begin{array}{c}\text { Porcentaje } \\
(\mathbf{\%})\end{array}$ & $\begin{array}{c}\text { Porcentaje } \\
\text { Acumulado } \\
(\mathbf{\%})\end{array}$ \\
\hline Regular & 2 & 4.3 & 4.3 \\
Buena & 12 & 26.1 & 30.4 \\
Muy Buena & 32 & 69.6 & 100.0 \\
Total & 46 & 100.0 & \\
\hline
\end{tabular}

Fuente: Elaboración propia
Figura 12. Distribución de 46 Estudiantes de la FAUA - UNI, según su Nivel de Actitudes de Aprender a Convivir

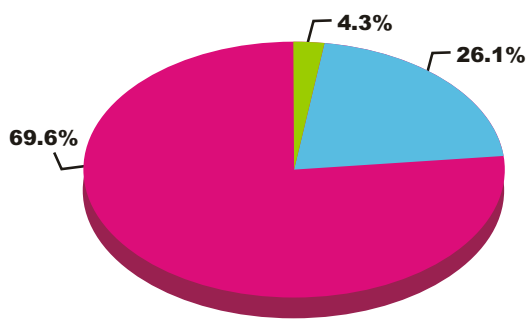

Regular Buena Muy Buena

Fuente: Elaboración propia

Respecto a los niveles de actitudes de aprender a convivir de los estudiantes de la FAUA de la Universidad Nacional de Ingeniería, se observa en la Tabla 12 y Figura 12 que el $69.6 \%$ de los encuestados consideran que su actitud es muy buena; el $26.1 \%$, buena, y el $4.3 \%$, regular.

Tabla 13. Distribución de 46 Estudiantes de la FAUA - UNI, según su Nivel de Actitudes de Comportamiento del Individuo con el Medio

\begin{tabular}{l|c|c|c}
\hline Niveles & Frecuencias & $\begin{array}{c}\text { Porcentaje } \\
\text { (\%) }\end{array}$ & $\begin{array}{c}\text { Porcentaje } \\
\text { Acumulado } \\
(\%)\end{array}$ \\
\hline Mala & 1 & 2.2 & 2.2 \\
Regular & 8 & 17.4 & 19.6 \\
Buena & 19 & 41.3 & 60.9 \\
Muy Buena & 18 & 39.1 & 100.0 \\
Total & 46 & 100.0 & \\
\hline
\end{tabular}

Fuente: Elaboración propia

Figura 13. Distribución de 46 Estudiantes de la Facultad de Arquitectura de la UNI, según su Nivel de Actitudes de Comportamiento del Individuo con el Medio

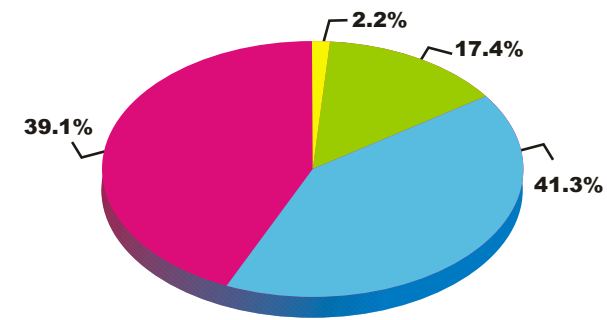

Mala Regular Buena Muy Buena

Fuente: Elaboración propia

Se observa en la Tabla 13 y Figura 13 que, respecto a sus niveles de actitudes de comportamiento del individuo con el medio de los estudiantes de la FAUA de la Universidad Nacional de Ingeniería, el $39.1 \%$ de los 
encuestados considera que es muy buena; el $41.3 \%$, buena; el $17.4 \%$, regular, y el $2.2 \%$, mala.

\section{Dimensión Personal}

Tabla 14. Distribución de 46 Estudiantes de la FAUA - UNI, según Nivel de Importancia del Aprendizaje Holístico Referido a lo Personal en la Formación Académica

\begin{tabular}{l|c|c|c}
\hline \multicolumn{1}{c|}{ Niveles } & Frecuencias & $\begin{array}{c}\text { Porcentaje } \\
\mathbf{( \% )}\end{array}$ & $\begin{array}{c}\text { Porcentaje } \\
\text { Acumulado } \\
(\mathbf{\%})\end{array}$ \\
\hline Mediana Importancia & 22 & 47.8 & 47.8 \\
Máxima Importancia & 24 & 52.2 & 100.0 \\
Total & 46 & 100.0 & \\
\hline
\end{tabular}

Fuente: Elaboración propia

Figura 14. Distribución de 46 Estudiantes de la FAUA - UNI, según Nivel de Importancia del Aprendizaje Holístico Referido a lo Personal en la Formación Académica

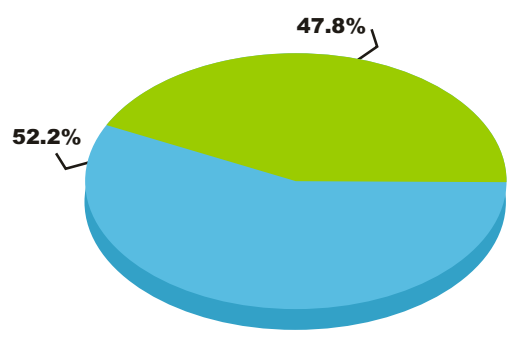

Mediana Importancia $\quad$ Máxima importancia

Fuente: Elaboración propia

En la Tabla 14 y Figura 14, respecto a los niveles de importancia sobre el aprendizaje holístico referido a lo personal en la formación académica de los estudiantes de la FAUA de la Universidad Nacional de Ingeniería, el 52.2\% de los encuestados considera que es de máxima importancia, mientras que el $47.8 \%$, de mediana importancia.

Tabla 15. Distribución de 46 Estudiantes de la FAUA - UNI, según Sí/No logra un Aprendizaje Holístico referido a Nuevos Conocimientos en la Formación Académica

\begin{tabular}{c|c|c|c}
\hline Niveles & Frecuencias & $\begin{array}{c}\text { Porcentaje } \\
(\%)\end{array}$ & $\begin{array}{c}\text { Porcentaje } \\
\text { Acumulado } \\
(\%)\end{array}$ \\
\hline No & 31 & 67.4 & 67.4 \\
Sí & 15 & 32.6 & 100.0 \\
Total & 46 & 100.0 & \\
\hline
\end{tabular}

Fuente: Elaboración propia

Figura 15. Distribución de 46 Estudiantes de la FAUA - UNI, según Sí/No Logra un Aprendizaje Holístico Referido a Nuevos Conocimientos en la Formación Académica

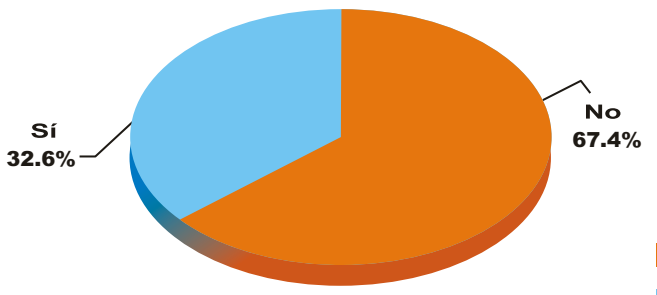

Fuente: Elaboración propia

No

si

La Tabla 15 y Figura 15 permite observar que el $32.6 \%$ de los estudiantes de la FAUA de la Universidad Nacional de Ingeniería considera que sí logra un aprendizaje holístico referido a nuevos conocimientos en su formación académica; el $67.4 \%$, no.

Tabla 16. Distribución de 46 Estudiantes de la FAUA - UNI, según Sí/No Logra un Aprendizaje Holístico Referido

al Desarrollo Físico, Cognitivo y Espiritual en la Formación Académica

\begin{tabular}{c|c|c|c}
\hline Niveles & Frecuencias & $\begin{array}{c}\text { Porcentaje } \\
(\mathbf{\%})\end{array}$ & $\begin{array}{c}\text { Porcentaje } \\
\text { Acumulado } \\
(\%)\end{array}$ \\
\hline No & 31 & 67.4 & 67.4 \\
Sí & 15 & 32.6 & 100.0 \\
Total & 46 & 100.0 & \\
\hline
\end{tabular}

Fuente: Elaboración propia

Figura 16. Distribución de 46 Estudiantes de la FAUA - UNI, según Sí/No Logra un Aprendizaje Holístico Referido al Desarrollo Físico, Cognitivo y Espiritual en la Formación Académica

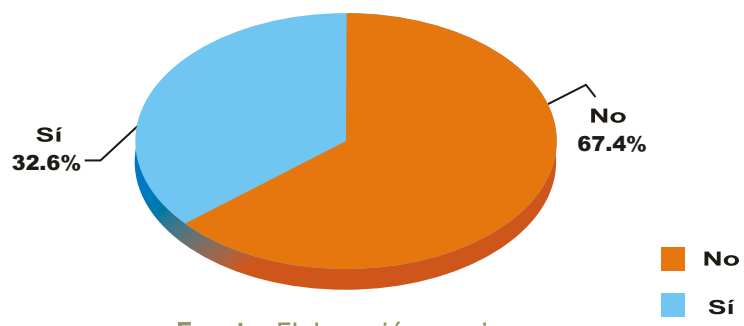

Fuente: Elaboración propia

Se observa de la Tabla 16 y Figura 16, que el $32.6 \%$, de los estudiantes de la Facultad de Arquitectura, Urbanismo y Artes de la Universidad Nacional de Ingeniería consideran que sí logran un Aprendizaje Holístico referido al Desarrollo Físico, Cognitivo y Espiritual; y el $67.4 \%$, consideran que no logran. 


\section{Dimensión Ambiental}

Tabla 17. Distribución de 46 Estudiantes de la FAUA - UNI, según Niveles de Aprendizaje Holístico Referido

a lo Ambiental en su Formación Académica

\begin{tabular}{l|c|c|c}
\hline Niveles & Frecuencias & $\begin{array}{c}\text { Porcentaje } \\
(\%)\end{array}$ & $\begin{array}{c}\text { Porcentaje } \\
\text { Acumulado } \\
(\%)\end{array}$ \\
\hline Malo & 1 & 2.2 & 2.2 \\
Regular & 6 & 13.0 & 15.2 \\
Bueno & 39 & 84.8 & 100.0 \\
Total & 46 & 100.0 & \\
\hline
\end{tabular}

Fuente: Elaboración propia

Figura 17. Distribución de 46 Estudiantes de la FAUA - UNI, según Niveles de Aprendizaje Holístico Referido a lo Ambiental en la Formación Académica

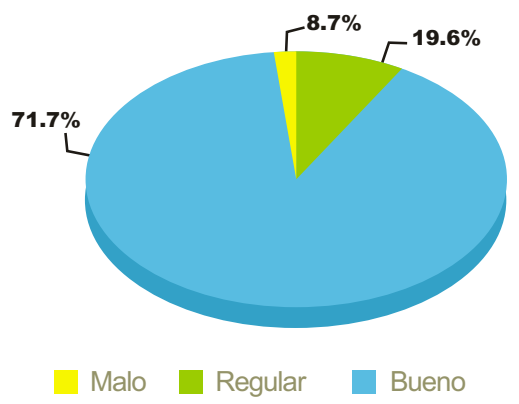

Fuente: Elaboración propia

Respecto a los niveles de Aprendizaje Holístico referido a lo Ambiental en su formación académica de los estudiantes de la FAUA de la Universidad Nacional de Ingeniería, se aprecia en la Tabla 17 y Figura 17 que el $84.8 \%$ de los encuestados considera que su nivel es bueno; el $13.0 \%$, regular, y el $2.2 \%$, mala.

Tabla 18. Distribución de 46 Estudiantes de la FAUA - UNI, según Niveles de Aprendizaje Holístico Referido a la Conciencia Ecológica en su Formación Académica

\begin{tabular}{l|c|c|c}
\hline Niveles & Frecuencias & $\begin{array}{c}\text { Porcentaje } \\
(\mathbf{\%})\end{array}$ & $\begin{array}{c}\text { Porcentaje } \\
\text { Acumulado } \\
(\mathbf{\%})\end{array}$ \\
\hline Muy Mala & 1 & 2.2 & 2.2 \\
Buena & 11 & 23.9 & 26.1 \\
Muy Buena & 34 & 93.9 & 100.0 \\
Total & 46 & 100.0 & \\
\hline
\end{tabular}

Fuente: Elaboración propia
Figura 18. Distribución de 46 Estudiantes de la FAUA - UNI, según Niveles de Aprendizaje Holístico Referido a la Conciencia Ambiental en su Formación Académica

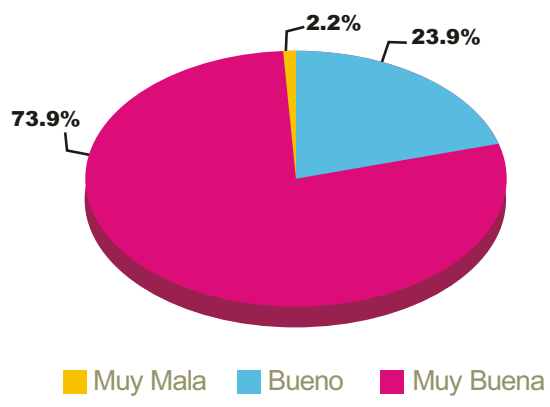

Fuente: Elaboración propia

En la Tabla 18 y Figura 18, se puede observar que, respecto a los niveles de aprendizaje holístico referido a la conciencia ambiental en su formación académica de los estudiantes de la FAUA de la Universidad Nacional de Ingeniería, el $73.9 \%$ de los encuestados considera que es muy bueno; el $23.9 \%$, bueno, yel $2.2 \%$, muy malo.

Tabla 19. Distribución de 46 Estudiantes de la FAUA - UNI, según Niveles de Aprendizaje Holístico Referido al Compromiso Social y Ciudadano en su Formación Académica

\begin{tabular}{l|c|c|c}
\hline Niveles & Frecuencias & $\begin{array}{c}\text { Porcentaje } \\
(\mathbf{\%})\end{array}$ & $\begin{array}{c}\text { Porcentaje } \\
\text { Acumulado } \\
(\%)\end{array}$ \\
\hline Muy Mala & 1 & 2.2 & 2.2 \\
Regular & 10 & 21.7 & 23.9 \\
Buena & 26 & 56.5 & 80.4 \\
Muy Buena & 9 & 19.1 & 100.0 \\
Total & 46 & 100.0 & \\
\hline
\end{tabular}

Fuente: Elaboración propia

Figura 19. Distribución de 46 Estudiantes de la FAUA - UNI, según Niveles de Aprendizaje Holístico Referido al Compromiso Social y Ciudadano en su Formación Académica

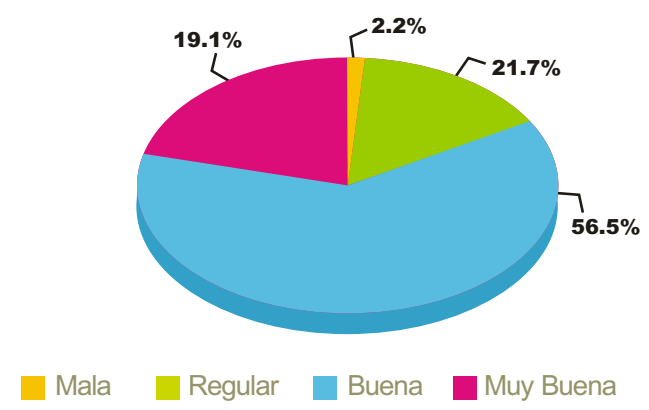

Fuente: Elaboración propia 
Se observa, en la Tabla 19 y Figura 19, respecto a los niveles de aprendizaje holístico referido al compromiso social y ciudadano en su formación académica de los estudiantes de la FAUA de la Universidad Nacional de Ingeniería, que el $19.6 \%$ de los encuestados considera que dicho nivel es muy bueno; el $56.5 \%$, bueno; el $21.7 \%$, regular, y el $2.2 \%$, muy malo.

\section{DISCUSIÓN}

Según los resultados expuestos, existe un alto porcentaje de docentes que consideran que sí es importante incorporar la educación ambiental para la sustentabilidad en la formación académica de los estudiantes. Asimismo, se ha determinado que los estudiantes consideran de máxima importancia recibir un aprendizaje holístico en su formación académica, lo cual incluye las dimensiones interpersonal (valores y actitudes), personal (nuevos conocimientos, desarrollo físico, cognitivo, espiritual y de satisfacción), ambiental (conciencia ambiental, compromiso social y ciudadano). De ahí que se puede afirmar que existe concordancia con lo que sostiene Gómez (2011):

Incorporar la educación ambiental al currículo plantea la conveniencia de ir más allá de los simples añadidos de temas ambientales a los contenidos, incluso más allá de la creación de algunas asignaturas o áreas específicamente dedicadas al tema ( $p$. 114).

Sin embargo, Cóndor (2016) sugiere, además, que "para lograr la sostenibilidad de la educación superior no es suficiente la formación ambiental de los estudiantes, también es importante trabajar en la docencia, investigación, extensión y proyección social” ( $p$. 137).
De otro lado, se coincide con lo que sostiene Espino (como se citó en Tax, 2014) cuando afirma que el aprendizaje forma parte de la visión holística, donde el ser humano declara las diferencias que ocurre en lo emocional, en lo espiritual, en lo artístico, en lo cognitivo y en lo espacial.

Los resultados estadísticos confirman que los docentes consideran que es muy importante incorporar la educación ambiental para la sustentabilidad como eje transversal en la formación académica de los estudiantes en la FAUA - UNI, con contenidos actitudinales, y axiológicos. Aunque, según los resultados, se evidencia que en menor escala lo incorporan en sus diferentes asignaturas. En cambio, los estudiantes consideran de máxima importancia el aprendizaje holístico en su formación académica, referido a lo interpersonal, es decir, en cuanto a las actitudes y comportamientos basados en valores. A partir de ello, se puede interpretar que la poca incorporación de educación ambiental en sus asignaturas se debe a que los docentes no tienen una formación adecuada en los temas ambientales a pesar de que destacan la importancia de incluir la dimensión ambiental en el plan curricular. Este resultado concuerda con lo que sostiene Cóndor (2016) cuando refiere que los docentes "tienen dificultades para incorporar la dimensión ambiental debido a que no tuvieron una formación adecuada en temáticas ambientales, por lo que es necesario realizar acciones de formación para incorporar la dimensión ambiental en dicha práctica" (p. 136).

Asimismo, los resultados determinan que para los docentes de la FAUA-UNI sí es importante incorporar la educación ambiental para la sustentabilidad como enfoque interdisciplinario en la formación académica de los estudiantes, y que para ello es importante la interacción de las diversas disciplinas; sin embargo, no hay evidencias estadísticas que afirmen que los estudiantes de la FAUA-UNI 
consideren que es muy importante lograr una transmisión activa de nuevos conocimientos, desarrollo físico cognitivo, espiritual y de satisfacción, por lo que hay un alto porcentaje de estudiantes que no logran tener un aprendizaje holístico.

Esto concuerda con lo que refiere Gonzáles y Giraldo (2014) cuando menciona que el cambio que se pide en la formación docente tiene que ver con la intencionalidad de la educación, y en especial de la educación ambiental, de transversalizar las diferentes materias, ponerlas en un mismo plano, donde se interrelacionan. Asimismo, estos autores plantean que "se debe replantear el diálogo vertical y compartimentado de las disciplinas, proponiendo nuevas dinámicas que impliquen el abordaje de problemáticas ambientales desde la transversalidad e interdisciplinariedad de las ciencias" (p. 198). No obstante, a pesar de que los docentes consideran la importancia de la interacción de diversas disciplinas en la formación académica de los estudiantes, esto no coincide con los resultados los cuales muestran la importancia que le otorgan los estudiantes al aprendizaje holístico en la dimensión personal, nuevos conocimientos, desarrollo físico, cognitivo, espiritual y de satisfacción. Ello tal vez se deba a un egocentrismo intelectual y al hermetismo del pensamiento que se protege para evitar que sea cuestionado o enriquecido desde otra perspectiva. Este resultado no concuerda con lo que establece Briceño et al. (2009) cuando refieren que el paradigma holístico es un camino para obtener conocimiento, en donde el ser humano estudia globalmente la interacción de sus manifestaciones espiritual, psíquica, orgánica, social y energética, tanto en lo interno como con sus semejantes y la naturaleza, para mantener el equilibrio armónico y funcional.

Por otro lado, según los resultados, para los docentes de la FAUA- UNI sí es importante la incorporación de la educación ambiental para la sustentabilidad como enfoque transdisciplinario en la formación académica de los estudiantes; es decir, con conocimiento global y multidimensional de los diversos aspectos de la realidad para lograr tener una conciencia ambiental, compromiso social y ciudadano. Esto último es de mucha importancia en los estudiantes. Lo señalado concuerda con Nicolescu (1998) quien menciona que la visión transdisciplinaria nos propone considerar una realidad multidimensional, estructurada a varios niveles, lo cual reemplaza la realidad unidimensional del pensamiento clásico. Ello quiere decir que una investigación transdisciplinaria analiza los diferentes niveles de la realidad a la vez, buscando comprender el mundo y llegando a la unidad del conocimiento.

También, a partir de los resultados, se coincide con Espejel, Castillo y Martínez (2011), para quienes la conciencia ambiental es el conjunto de vivencias, conocimientos, percepciones, actitudes, conductas, valores, motivaciones y experiencias que el individuo utiliza activamente para solucionar de forma sustentable problemas de su ambiente.

\section{CONCLUSIONES}

Se concluye que hay evidencias estadísticas para afirmar que la educación ambiental para la sustentabilidad es importante en la formación académica estudiantil para un aprendizaje con visión holística, basado en la integración de conocimientos, en la FAUA - UNI. Esto se evidencia con los resultados de las encuestas donde el $94.4 \%$ de los docentes de la FAUA consideran que sí es importante la incorporación de esta línea temática en la formación académica de los estudiantes de esta Facultad. Y en cuanto a los niveles de importancia respecto al aprendizaje holístico de los estudiantes de la FAUA - UNI, el 78.3\% de los encuestados consideran que este aprendizaje es de máxima importancia, y el $21.7 \%$, de mediana importancia. 
Así también, se concluye que hay evidencias estadísticas para afirmar que los docentes, en menor escala, incorporan, en sus diferentes asignaturas, la educación ambiental con contenidos actitudinales y axiológicos que contribuyan en la formación de valores y actitudes de convivencia, respeto y de comportamiento del individuo con el medio en la Facultad de Arquitectura, Urbanismo y Artes de la Universidad Nacional de Ingeniería.

Otro dato importante que se observa en los resultados de las encuestas es que un $94.4 \%$, de los docentes de la FAUA-UNI considera importante incorporar la educación ambiental para la sustentabilidad como eje transversal en la formación académica de los estudiantes de esta Facultad; frente a un $5.6 \%$ de encuestados que no lo considera así. Por otro lado, en cuanto a los niveles de importancia respecto al aprendizaje holístico referido a lo interpersonal en la formación académica de los estudiantes de la FAUA-UNI, se le otorga una importancia máxima (78.3\%) y una mediana (21.7\%).

También las evidencias estadísticas determinan que los docentes de FAUA-UNI consideran importante la interacción de diversas disciplinas (enfoque interdisciplinario) en la formación académica estudiantil. Sin embargo, no hay evidencias estadísticas para afirmar que los estudiantes de la FAUA-UNI consideran que es muy importante lograr una transmisión activa de nuevos conocimientos, desarrollo físico, cognitivo, espiritual y de satisfacción (personal).

Además, los resultados también permiten observar que el $7.2 \%$ de los docentes de la FAUA - UNI considera importante la incorporación de la educación ambiental para la sustentabilidad como enfoque interdisciplinario en la formación académica de los estudiantes de esta Facultad; frente a quienes manifiestan lo contrario (2.8\%). Asimismo, en cuanto a los niveles de importancia respecto al aprendizaje holístico referido a lo personal en la formación académica de los estudiantes de la FAUA- UNI, el $52.2 \%$ de los encuestados considera que es de máxima importancia, y el $47.8 \%$, de mediana importancia.

Las evidencias estadísticas afirman que los estudiantes de la FAUA-UNI consideran que es muy importante tener una conciencia ambiental, compromiso social y ciudadano, así como los docentes afirman la importancia del conocimiento global y multidimensional (enfoque transdisciplinario) en la formación académica estudiantil. De las dos afirmaciones anteriores se concluye que hay evidencias estadísticas para afirmar que los docentes consideran que es importante el conocimiento global y multidimensional de los diversos aspectos de la realidad en la formación académica estudiantil para lograr tener una conciencia ambiental, y de compromiso social y ciudadano.

\section{REFERENCIAS BIBLIOGRÁFICAS}

Briceño, J., Cañizales, B., Rivas, Y., Lobo, H., Moreno, E., Velásquez, I. y Ruzza, I. (2009). La holística y su articulación con la generación de teorías. Educere, 14(48), 73-83.

Cóndor, E. (2016). Dimensión ambiental en la formación profesional de los estudiantes de la Facultad de Educación de la Universidad Nacional de Huancavelica [Tesis de doctorado, Universidad Nacional del Centro del Perú]. Repositorio Institucional.

Esquerra, G., Gil, J. y Márquez, F. (2016). Educación para el desarrollo sostenible, su dimensión ambiental. Una visión desde y para las universidades en América Latina. Revista Estudios del Desarrollo Social: Cuba y América Latina, (4), 72-81.

http://scielo.sld.cu/pdf/reds/v4n3/reds0 7316.pdf 
Espejel, A., Castillo, I. y Martínez, H. (2011). Modelo de educación ambiental para el nivel medio superior, en la región Puebla - Tlaxcala, México: un enfoque por competencias. Iberoamericana de Educación, 55(4), 1-13. https://doi.org/10.35362/rie5541584

Gómez, C. (2011). La educación ambiental en la formación superior en Medellín: estudio de caso en dos niveles de tres instituciones [Tesis de maestría, Universidad Nacional de Colombia]. Repositorio Institucional.

Gonzáles, V. y Giraldo, Y. (2014). Educación ambiental y extensión universitaria: una mirada crítica del currículo [Tesis de licenciatura, Universidad de Antioquía]. Repositorio Institucional.

Huamaní, C. (2017). Eficacia del método de proyectos en el aprendizaje de la educación ambiental de los alumnos de la Facultad de Educación de la Universidad Nacional San Cristóbal de Huamanga de Ayacucho, Perú en el año 2013 [Tesis de maestría, Universidad Nacional de Educación Enrique Guzmán y Valle]. Repositorio Institucional.

Molano, A. (2013). Concepciones y prácticas sobre educación ambiental de los docentes en las universidades de Bogotá. Implicaciones para los currículos de las facultades de educación [Tesis de doctorado, Universidad de Valladolid]. Repositorio Institucional.

Molano, A. y Herrera, J. (2014). La Formación ambiental en la educación superior: una revisión necesaria. Luna Azul, 39, 1-21. http://www.scielo.org.co/pdf/luaz/n39/ n39a12.pdf

Nicolescu, B. (1998). La transdisciplinariedad, una nueva visión del mundo. Manifiesto. Ediciones Du Rocher.

Pernía, J. (2016). Orientaciones educativas ambientales para un modelo de universidad sustentable en la UCAB a partir de su comunidad de aprendizaje [Tesis de doctorado, Universidad Católica Andrés Bello]. Repositorio Institucional.

Tax, E. (2014). Método holístico y aprendizaje de ecuaciones cuadráticas [Tesis de licenciatura, Universidad Rafael Landívar]. Repositorio Institucional.

Velásquez, J. (2009). La transversalidad como posibilidad curricular desde la educación ambiental. Revista Latinoamericana de Estudios Educativos, 5 (2), 29-44.

http://www.redalyc.org/pdf/1341/134116861 003.pdf 